\title{
EDITORIAL
}

\section{Apoptotic cell clearance and fibrotic lung disease}

\author{
William J. Janssen* and Konosuke Morimoto\#
}

$\mathbf{R}$ ecognition and clearance of dying cells by phagocytes (a process known as efferocytosis) is essential for development, tissue homeostasis, and recovery from inflammation and injury. Under most circumstances, this process is so efficient that it can be difficult to detect, as illustrated by the fact that $>1 \times 10^{11}$ neutrophils are eliminated from the human circulation each day in a process that leaves no obvious trace [1]. Along similar lines, during communityacquired pneumonia there may be $>1.1 \times 10^{6}$ neutrophils per cubic millimetre of lung tissue, yet clearance of dying cells is so efficient that $<0.3 \%$ of the neutrophils in the lungs are apoptotic [2]. While the consequences of apoptotic cell removal were initially thought to be immunologically silent, it is now recognised that efferocytosis can lead to the production of potent anti-inflammatory molecules, such as transforming growth factor (TGF)- $\beta$ and interleukin-10, and growth factors that enhance tissue repair, such as hepatocyte growth factor (HGF) and vascular endothelial growth factor [3-5]. It is not surprising then that intentional injection of apoptotic cells into inflamed lungs or peritonea can enhance resolution of inflammation. In this issue of the European Respiratory Journal, LEE et al. [6] build on this foundation by demonstrating that a single administration of apoptotic cells into the lungs of bleomycin-injured mice reduces acute inflammation, attenuates activation of proapoptotic caspases and ameliorates the development of fibrosis. These striking results raise two important questions. How are these effects achieved and can these results be applied to human lung disease?

In answer to the first question, the results presented by LeE et al. [6] implicate HGF as a key molecule. Their results show that instillation of apoptotic cells into the lungs leads to an immediate increase in HGF gene expression and protein levels in the lungs. These findings are in line with previous work that shows that efferocytosis induces HGF production by macrophages $[5,7]$. Accordingly, there is strong evidence suggesting that HGF upregulates antiapoptotic signalling pathways in epithelial and endothelial cells, rendering them less susceptible to oxidant and Fas-mediated apoptosis [8], both of which have been implicated in bleomycin-induced lung injury. Since epithelial injury is believed to be a prerequisite for the development of fibrosis, it is not surprising then that administration of HGF (either directly into the lungs or through systemic routes) attenuates fibrosis in bleomycin-treated rodents.

\footnotetext{
*National Jewish Health, Denver, CO, USA. ${ }^{\#}$ Dept of Clinical Medicine, Institute of Tropical Medicine, Nagasaki University, Nagasaki, Japan.

CORRESPONDENCE: K. Morimoto, Dept of Clinical Medicine, Institute of Tropical Medicine, Nagasaki University, 1-12-4, Sakamoto, Nagasaki, 852-8523, Japan. E-mail: komorimo@nagasaki-u.ac.jp
}

An interesting aspect of the work presented by LEE et al. [6] is that a single instillation of apoptotic cells resulted in enhanced expression of HGF in the lungs for up to 21 days. The mechanisms underlying this effect are unknown; however, it is enticing to postulate that ingestion of apoptotic cells altered alveolar macrophage activation states, a factor known to be important in fibrogenesis [9]. Along similar lines, it is important to note that instillation of apoptotic cells by LEE et al. [6] resulted in early, but not sustained, increases in TGF- $\beta$. This result may be considered ideal, since during acute inflammation, TGF exerts anti-inflammatory effects, whereas sustained expression of TGF- $\beta$ in the lungs is generally regarded as pro-fibrotic. In this context, a balance between HGF and TGF- $\beta$ may be beneficial to prevent epithelial apoptosis and promote repair of injured tissues without inducing fibrosis. Elegant studies from the liver and kidneys serve as an example for this paradigm wherein during resolution of inflammation, HGF suppresses TGF- $\beta$ production and mitigates fibrosis [10-12].

Administration of exogenous HGF was once considered a promising therapeutic strategy for pulmonary fibrosis [13]. Unfortunately, the molecule is too labile in the circulation to maintain suitable levels for long periods and perform antifibrotic actions. As LEE et al. [6] show, administration of apoptotic cells may be a more favourable means of inducing HGF in lungs, since the effect appears to be sustained. Moreover, efferocytosis of apoptotic cells may lead to immune modulation, including regulation of TGF- $\beta$. While these effects may be important in animal models of fibrosis, it is unclear whether similar efficacy can be realised in idiopathic pulmonary fibrosis (IPF). Most patients with this disease have established fibrosis at the time of presentation and it is unlikely that administration of apoptotic cells would lead to disease reversal. Furthermore, the role of inflammation in the progression of IPF is unclear and anti-inflammatory therapies have shown disappointing results. Thus, it is overly optimistic to assume that administration of apoptotic cells might have similar efficacy in humans with IPF. With this in mind, it is important to note that the bleomycin model of lung injury also mimics many features of acute lung injury and the acute respiratory distress syndrome. Might it therefore be possible to administer autologous apoptotic cells to patients with acute inflammatory lung injury? More research is required to determine whether this is feasible or even prudent. In addition to further understanding the biological mechanisms by which efferocytosis modulates immunity, it would be important to determine a number of factors, including the appropriate route of delivery for cells, the optimum volume and concentration of cells, the frequency of treatment, the best type of cells to use and the best way to induce apoptosis. 


\section{STATEMENT OF INTEREST}

None declared.

\section{REFERENCES}

1 Stark MA, Huo Y, Burcin TL, et al. Phagocytosis of apoptotic neutrophils regulates granulopoiesis via IL-23 and IL-17. Immunity 2005; 22: 285-294.

2 Droemann D, Aries SP, Hansen F, et al. Decreased apoptosis and increased activation of alveolar neutrophils in bacterial pneumonia. Chest 2000; 117: 1679-1684.

3 Fadok VA, Bratton DL, Konowal A, et al. Macrophages that have ingested apoptotic cells in vitro inhibit proinflammatory cytokine production through autocrine/paracrine mechanisms involving TGF- $\beta$, PGE2, and PAF. J Clin Invest 1998; 101: 890-898.

4 Golpon HA, Fadok VA, Taraseviciene-Stewart L, et al. Life after corpse engulfment: phagocytosis of apoptotic cells leads to VEGF secretion and cell growth. FASEB J 2004; 18: 1716-1718.

5 Morimoto K, Amano H, Sonoda F, et al. Alveolar macrophages that phagocytose apoptotic neutrophils produce hepatocyte growth factor during bacterial pneumonia in mice. Am J Respir Cell Mol Biol 2001; 24: 608-615.
6 Lee Y-J, Moon C, Lee SH, et al. Apoptotic cell instillation after bleomycin attenuates lung injury through hepatocyte growth factor induction. Eur Respir J 2012; 40: 424-435.

7 Park HJ, Choi YH, Cho YJ, et al. RhoA-mediated signaling upregulates hepatocyte growth factor gene and protein expression in response to apoptotic cells. J Leukoc Biol 2011; 89: 399-411.

8 Wang X, Zhou Y, Kim HP, et al. Hepatocyte growth factor protects against hypoxia/reoxygenation-induced apoptosis in endothelial cells. J Biol Chem 2004; 279: 5237-5243.

9 Gordon S. Alternative activation of macrophages. Nat Rev Immunol 2003; 3: 23-35.

10 Mizuno S, Nakamura T. Suppressions of chronic glomerular injuries and TGF- $\beta 1$ production by HGF in attenuation of murine diabetic nephropathy. Am J Physiol 2004; 286: F134-F143.

11 Xia JL, Dai C, Michalopoulos GK, et al. Hepatocyte growth factor attenuates liver fibrosis induced by bile duct ligation. Am J Pathol 2006; 168: 1500-1512.

12 Yang J, Dai C, Liu Y. Systemic administration of naked plasmid encoding hepatocyte growth factor ameliorates chronic renal fibrosis in mice. Gene Ther 2001; 8: 1470-1479.

13 Yaekashiwa M, Nakayama S, Ohnuma K, et al. Simultaneous or delayed administration of hepatocyte growth factor equally represses the fibrotic changes in murine lung injury induced by bleomycin. A morphologic study. Am J Respir Crit Care Med 1997; 156: 1937-1944. 